\title{
eJRIEPS
}

Ejournal de la recherche sur l'intervention en éducation physique et sport

42 | 2017

Varia

\section{Transitions codiques, charge sémique et contrat didactique en EPS}

Codiques transitions, semique load and didactic contract in EPS

\section{Gilles Marrot}

\section{(2) OpenEdition \\ Journals}

Édition électronique

URL : http://journals.openedition.org/ejrieps/555

DOI : 10.4000/ejrieps.555

ISSN : 2105-0821

Éditeur

ELLIADD

\section{Référence électronique}

Gilles Marrot, "Transitions codiques, charge sémique et contrat didactique en EPS », eJRIEPS [En ligne], 42 | 2017, mis en ligne le 01 juillet 2017, consulté le 01 août 2019. URL : http:// journals.openedition.org/ejrieps/555 ; DOI : 10.4000/ejrieps.555

\section{(c)}

La revue eJRIEPS est mise à disposition selon les termes de la Creative Commons Attribution 4.0 International License. 
eJRIEPS 42 juillet 2017

Transitions codiques, charge sémique et contrat didactique en EPS

Gilles Marrot

Université des Antilles, CRREF (EA 4538)

Résumé

Cet article veut rendre compte d'un travail de recherche inscrit dans le champ de la didactique de l'EPS. II propose d'interroger l'acte d'intervention du professeur d'EPS en étudiant la manière dont il identifie, sélectionne et articule les différents canaux de communication pour interagir avec sa classe afin d'y faire progresser ses élèves. Inscrite dans la veine théorique de la théorie de l'action conjointe en didactique, cette étude cible la dynamique des ajustements communicationnels qui s'exercent entre ces deux acteurs du processus d'enseignement/apprentissage, et explore l'émergence des phénomènes de contrat didactique différentiel et de trilogue. La finalité de cette présentation consiste à poser les jalons d'un travail d'analyse et d'identification du processus de construction de compétences professionnelles relatives à la conduite de l'interaction dans l'intervention.

Mots-clés : intervention, interaction, communication, contrat didactique, transition codique, gestes professionnels.

\section{Introduction}

L'agir enseignant, dans le cadre de situations professionnelles authentiques visant la construction par les élèves de contenus, est piloté par une compétence à pianoter sur un clavier assez large de canaux de communication. Dans une perspective interactionniste, la notion de multicanalité (Barrière-Boizumault, 2013, p. 9) désigne ce phénomène. Dans bien des cas, la mise en oeuvre de cette compétence semble relever d'un tâtonnement intuitif : lorsque le canal dominant initial se révèle peu productif, l'enseignant en privilégie un autre qui, s'il ne produit pas les effets voulus, l'incite à en choisir un autre...puis un autre...jusqu'à ce qu'il trouve "le bon canal» ou le bon dosage entre tous les canaux. Cependant, ce changement peut aussi révéler une logique pédagogique préprogrammée et stabilisée, comme par exemple des consignes verbales adressées à tout le groupe en phase de définition de la tâche, des silences en phase de dévolution, des consignes gestuelles et verbales en phase de régulation. Ces phénomènes font-ils vraiment progresser les élèves ? Tous les élèves ? Sont-ils maîtrisables et donc perfectibles ? Peuvent-ils constituer un outil 


\section{eJRIEPS 42 juillet 2017}

de différenciation efficace? Nous ciblons là une difficulté professionnelle cruciale dans le processus de transmission des savoirs: l'activité adaptative de l'enseignant, décisionnelle ou énactive, quant aux modalités d'interaction auxquelles il peut avoir recours pour mieux aider les élèves dans leurs apprentissages.

Or, les dispositifs proposés aux élèves sont structurellement constitués de consignes, différentes en nature et en fonction (Famose, 1983). Alors comment marquer cette différence dans leur énonciation? Quel canal serait le plus approprié ? La gamme communicationnelle se complexifie donc au cœur des tâches et crée des conditions de réalisation différenciées : le milieu didactique en est donc affecté, et, si «l'actant agit en fonction d'un milieu » (Brousseau, 2004, p.4) c'est également le cas pour l'activité des élèves. Y a-t-il alors des canaux ou des enchainements de canaux de communication à privilégier pour obtenir une avancée des savoirs sur l'axe du temps?

Les interactions communicationnelles, caractérisant le milieu didactique et orientant l'activité de l'élève, vont aussi se spécifier au regard des ressources disponibles, des modes d'apprentissage, du niveau de développement, du capital culturel, des faisceaux de préoccupations de ce dernier. La prise en compte de ce « déjà là » (Astolfi \& Develay, 1989) interroge donc le registre communicationnel à activer pour obtenir les transformations recherchées. Mais ces ressources que l'élève va mobiliser dépendent également des contraintes intrinsèques aux activités physiques sportives et artistiques (APSA) qui fondent leurs enjeux fondamentaux et spécifiques. Certains modes d'intervention et de communication seront-ils donc à privilégier en fonction des APSA support proposées ?

Cette recherche vise à explorer, dans la complexité de l'interaction didactique en EPS, comment l'enseignant va orchestrer et exploiter cette multi-canalité au service des apprentissages des élèves. Elle interroge donc le processus de construction de compétences professionnelles sur ce thème.

\section{Cadre théorique}

2 .1. Communications verbales, non verbales, coverbales.

Dans une perspective interactionniste, (qui envisage la construction d'un phénomène à partir de sa constante redéfinition de ses rapports à son environnement), l'acte d'enseignement ne se conçoit plus sans prendre en compte les processus d'interactions qui organisent la transmission de connaissances en contexte situé (Durand, 2001 ; Mahut et al., 2005 ; Broussal \& Bucheton, 2008 ; Boudard \& Robin, 2012). II relève de la mise en œuvre de compétences du métier dont les gestes professionnels constituent des indicateurs (Brière- 


\section{eJRIEPS 42 juillet 2017}

Guenoun, 2014) et sur lesquels portent certains travaux (Roncin \& Loquet, 2007 ; Chevallard, 1997 ; Roesslé \& Loquet, 2005). Ces derniers étudient ce que met en œuvre l'enseignant pour transmettre les contenus d'enseignement qu'il a sélectionnés. La communication est un de ces éléments. Nous en retiendrons trois catégories : la communication verbale ou langage verbal (Broussal \& Bucheton, 2008 ; Bataille, 2011 ; Trottin \& Cogerino, 2009), la communication non verbale (Moulin, 2004 ; Boizumault \& Cogerino, 2012) et la communication coverbale (Mahut, 2003 ; Mc Neill, 1992 ; Cosnier \& Brossard, 1984). Nous entendrons par «communication verbale » ce que l'enseignant énonce comme consignes audibles par l'élève, qui en retour va interpréter ce message pour ensuite le traduire en comportement moteur, méthodologique ou social. La « communication non verbale » (CNV) désigne les « échanges supportés par les évènements cinétiques lents et surtout rapides : posturo-mimo-gestualité, eux-mêmes de plus en plus intégrés dans la communication totale aux côtés des activités vocales et verbales » (Cosnier \& Brossard, 1984). La «communication coverbale» désigne le répertoire gestuel convoqué pour contribuer à la partie verbale de l'interaction avec mises en jeu des attitudes. Ce « langage » prend le qualificatif de «gestualité syllinguistique »(Anzieu \& Cosnier, 1982), ou encore gestuelle co-verbale. Par définition il coexiste avec le langage parlé en lui étant concomitant (rapport au temps), ou associé ou en s'y substituant en partie. La gestuelle de l'enseignant, brute ou accompagnant le verbe, sera limitée dans notre étude, en EPS, à leur nature iconique en référence à la classification de Mahut (2003 p. 128). Nous y distinguerons les gestes «spatiographiques» (gestes représentant une relation spatiale) et les gestes «kinétographiques » (gestes représentant une action à faire). Ces choix sont motivés par deux raisons. D'une part car ils appartiennent à un code faible et donc peuvent rendre compte d'une pré-construction immuable et récurrente ou d'une co-construction instable et émergeante. D'autre part car ce sont deux catégories de gestes qui peuvent incontestablement se retrouver dans la logique d'étayage et de tissage (geste ayant pour fonction de piloter les apprentissages) (Mahut 2003, p. 144).

Parmi les CNV indicateurs de l'agir enseignant, nous accorderons un statut particulier « au silence » (Evin et al., 2013), qualifié souvent d'« indispensable » (Pain, 2012). II caractérise la « réticence didactique » (Sensevy \& Quilio, 2002 ; Sensevy, 2001), moment où le professeur devra « tenir par devers lui certaines des choses qu'il veut enseigner et à engager les élèves dans des rapports aux milieux qui leur permettront de passer outre... » (Sensevy \& Quilio, 2002, p. 50), mais aussi les actions de dévolution. Le silence organise des ruptures de l'attention favorisant l'observation efficace et la prise de décision (Djiksteruis \& Nordgren, 


\section{eJRIEPS 42 juillet 2017}

2006) par focalisation de la charge attentionnelle (attention sélective et attention exécutive) sur les éléments comportementaux des conduites au détriment du discours à produire. Dans le cours de l'intervention ou de l'expérience professionnelle, on peut concevoir que l'enseignant, qui aura intégré un certain nombre de conduites typiques et leur interprétation, réduira les moments de silence pour concéder de la place aux canaux de communication les plus pertinents. Cette gestion des silences, contributive de la multi-canalité communicationnelle, peut alors être interrogée: est-elle le fruit d'une réélaboration permanente au sein de chaque classe ou une compétence professionnelle généralisable à construire?

Enfin, parmi les CNV, nous inclurons «le déplacement " comme indicateur des gestes professionnels, car il scande le rapport au temps didactique en même temps qu'il constitue une CNV de nature à rendre compte de la dynamique des échanges communicationnels entre enseignant et élèves. II est le garant du maintien ou de la rupture du contrat de communication entre les inter-actants.

Ici se dessinent les contours de notre objet d'étude: l'enseignant, ayant pour "préoccupations » (Saujat, 2004) de transmettre des contenus d'enseignement à ses élèves, va développer une série de communications verbales, non verbales et coverbales qui sont indissociablement articulées pour en conserver toute la charge sémique vectrice d'efficacité dans l'apprentissage et donc garante de l'avancée des savoirs. Nous appellerons «transitions codiques" les moments précis marquant ce passage d'un canal de communication à un autre. Nous tenterons d'explorer les conditions ou les déterminants de ces transitions dans la discipline EPS. La matrice disciplinaire, où «le corps, la motricité, l'action et l'engagement de soi est au cœur des apprentissages " (Programmes d'EPS 2008), ceux-ci étant associés à «la nécessaire médiation par le langage oral et écrit » (programme d'EPS 2015), va-t-elle particulariser la nature des interactions langagières ? La hiérarchie illégitime (Barrière-Boisumault, 2013) du langage verbal, propre à beaucoup de disciplines scolaires et renforcée aujourd'hui par les programmes va-t-elle se confirmer ? Les dimensions sociales, méthodologiques et motrices des apprentissages ne vont-elles pas spécifier ces articulations communicationnelles ?

2. 2. La théorie de l'action conjointe en didactique comme champ théorique de référence pour comprendre les interactions de communication en EPS Les concepts que nous allons utiliser pour étudier l'action de l'enseignant sous l'angle de l'articulation de différents canaux de communication ainsi que ses effets sur l'activité d'apprentissage des élèves, sont empruntés à des domaines de recherche différents. Nous 


\section{eJRIEPS 42 juillet 2017}

tenterons de les mettre en convergence autour d'un faisceau de notions essentiellement empruntées à la théorie de l'action conjointe en didactique (TACD), qui constituera le champ dominant pour notre étude. En effet, la définition de notre objet prend racine au sein de l'interaction contractualisée entre un enseignant qui donne des consignes sous des formes variées et des élèves qui tentent de les décoder, pour construire des réponses motrices élaborées. On comprend alors que va s'instaurer une dynamique d'ajustement réciproque pilotant les changements de modes communicationnels de chacun des interactants.

Nous utiliserons d'abord la notion de «contrat didactique», entendue comme «un système d'habitus ou un système de règles du jeu immanent à telle ou telle situation, dont les implications réciproques sont constamment redéfinies dans l'action 》 (Brousseau, 1998). Nous la considèrerons indissociable du « milieu didactique », défini comme «'ensemble des objets de savoirs générateurs de ressources et de contraintes» (Rilhac, 2007). La TACD propose de modéliser la dynamique évolutive de ce doublet contrat-milieu didactique (Schubauer-Leoni \& Leutenegger, 2002 ; Sensevy \& Mercier, 2007) à partir de trois descripteurs : la mésogenèse, la topogenèse et la chronogenèse, « catégories articulées qui sont postulées comme génériques»(Sensevy 2007). Plusieurs publications en didactique des APSA en montrent la fonctionnalité pour notre étude (Amade-Escot, Verscheure \& Devos-Prieur, 2002 ; Loquet, Garnier \& Amade-Escot, 2002 ; Loquet, Roessle \& Roncin, 2007). Les travaux de Ligozat (2008) expliquent que ce triplet constitue un outil permettant de rendre compte des phénomènes didactiques à différents niveaux: microscopique, mésoscopique et macroscopique. Nous plaçons nos travaux à l'articulation des échelles mésodidactiques et microdidactiques ce qui nous permettra de rendre compte quantitativement et qualitativement des phénomènes. Certaines études ciblent également le contexte communicationnel pour réalimenter le regard porté sur les trois catégories de genèse (Leutenegger, 2008). II s'agit d'envisager les interactions enseignant/élève en terme de positionnement des élèves dans un système plus vaste que constituent le groupe classe et les sous groupes, ainsi que les échanges polylogaux dissymétriques représentés par le professeur, les élèves en position de locuteurs légitimes et autorisés et le reste de la classe. Cette approche est au cœur de nos préoccupations en EPS puisque les élèves se trouvent dans des groupes différents et que l'enseignant diversifie les registres de locution pour se faire comprendre, tout en multipliant les échanges avec chacun des groupes et avec certains élèves dans ces groupes. Pour le descripteur mésogénétique, qui implique un objet de transmission symbolique ou langagier, l'intervention gestuelle ou verbale de l'enseignant sera retenue. Pour le descripteur chronogénétique, qui rend compte de l'avancée 


\section{eJRIEPS 42 juillet 2017}

temporalisée des savoirs, nous regarderons les contenus d'enseignement, leur distribution dans le temps, leur conception, leur hiérarchisation. Le descripteur topogénétique sera examiné à partir du partage des responsabilités dans les interactions. Nous considèrerons que la chronogenèse se déroule de façon différenciée puisque chaque élève apprend « selon son propre rythme ». Si la question des savoirs permet à ce dernier d'établir un rapport particulier aux objets du milieu didactique, la mésogénèse ne peut que se différencier également. Le rapport au savoir évoluant de façon différenciée, les statuts des élèves au sein du groupe peuvent être amenés à évoluer, certains élèves dits chronogènes vont se manifester, ce qui va modifier la topogénèse. Ce mécanisme provoque la production de contrats didactiques différentiels, dans la mesure où se créent des dynamiques singulières via la différenciation langagière entre le professeur et des sous-groupes d'élèves ; il amène également à s'interroger sur la notion de trilogue (Schubauer-Leoni, 1997), traduisant le fait qu'un enseignant qui s'adresse à un élève interagit également avec des sous groupes d'élèves, voire avec le reste de la classe.

Nous exploiterons également les quatre actions didactiques de l'enseignant que la TACD (Sensevy \& Mercier, 2007) répertorie: la définition, la dévolution, la régulation, l'institutionnalisation.

La troisième notion que nous utiliserons est celle d'épistémologie pratique de l'enseignant, qui renvoie à ses conceptions de l'apprentissage, de l'enseignement, de l'élève, de la connaissance, des contenus d'enseignement... Nous étudierons dans quelle mesure ces ensembles de conceptions déterminent et motivent les articulations des modalités communicatives.

Nous retiendrons enfin l'idée de construction des tâches, l'intervention de l'enseignant se différenciant en fonction du type de situation qu'il propose.

\section{Problématique et hypothèses}

Si l'enseignement en EPS vise des apprentissages moteurs, et que la relation enseigner apprendre se détermine par des processus d'apprentissage induisant la priorisation de certains procédés d'enseignement, quelle place va être accordée aux différents canaux de communications en tant que voies de transmission de contenus, et comment ceux-ci s'articulent-ils les uns aux autres ? Lequel sera privilégié ? Comment la conception de l'APSA support des enseignements et de ses contenus intervient-elle dans la construction de cette articulation ? Comment évolue cette combinaison au fil des situations, des leçons et des relations avec les élèves? Quels sont les facteurs identifiables de cette évolution ? 


\section{eJRIEPS 42 juillet 2017}

Notre étude visera à montrer que la mise en synergie du gestuel/verbal/coverbal dépend d'une coconstruction liée aux écarts de conditions de production/réception des messages verbaux plus que d'une anticipation de leur production à des fins d'apprentissage ; autrement dit, l'articulation des procédés de transmission renvoie davantage à une dynamique située coconstruisant l'interaction enseignement/apprentissage qu'à une intervention planifiée.

Nous formulerons deux hypothèses. Premièrement, l'articulation des registres d'interaction utilisés avec les élèves est modélisable et quantifiable. Elle peut rendre compte de la logique quantitative et qualitative du contrat-milieu didactique différentiel. Deuxièmement, l'articulation des registres de transmission et sa dynamique d'évolution sont conditionnées davantage par les savoirs professionnels présidant à l'élaboration des contenus d'enseignement que par des évènements situationnels émergeants de l'interaction.

\section{Méthodologie}

Nous avons retenu pour notre recherche la natation comme activité physique support car les interactions et communications verbales enseignant/élève sont spécifiques. En effet, les ressources informationnelles habituellement disponibles chez l'élève "terrien" pour apprendre deviennent erronées par la mise en œuvre de la poussée d'Archimède et la densité du milieu. Le sens kinesthésique est faussé, la vue est troublée, l'élève ne peut entendre et nager simultanément. La démarche explicative et langagière usuelle dans bon nombre d'APSA se trouve donc contrariée, car écouter pour l'élève signifie l'arrêt de l'activité ou le redressement de la tête (et donc l'augmentation du maitre couple).Un re-calibrage modalitaire et intermodalitaire des informations sensorielles est alors requis pour augmenter la justesse des interactions avec cet environnement spécifique et créer les conditions d'une capture adéquate des consignes. Dans le cadre scolaire (Magne \& Schmitt, 2002), les contenus d'enseignement en natation et leur transmission dépendent donc d'abord des ressources disponibles ou rendues disponibles chez l'élève, mais aussi de la conception de l'activité (utilitaire ou sportive) (Pelayo, 1999 ; Gal, 1993 ; Catteau, 1992), du niveau scolaire (classement dans le champ 2 en cycle 3 et dans le champ 1 en cycle 4). La distance séparant l'enseignant de l'élève, la dissymétrie entre l'espace d'intervention et celui des apprentissages, du point de vue physique (l'enseignant et l'élève ne sont pas dans le même milieu), du point de vue du référentiel, (l'enseignant et l'élève ne sont pas dans la même position orthostatique), du point de vue du rapport au corps (l'un est habillé l'autre est presque nu) constituent le fondement structurel et fonctionnel du contrat didactique. Enfin, la sécurité (note de service, 1994 ; circulaire de 2004 ; circulaire de juillet 2011), engageant la 


\section{eJRIEPS 42 juillet 2017}

responsabilité de l'enseignant, génère chez ce dernier une triple contradiction : il est tiraillé entre la nécessité de faire avancer le savoir, celle de maintenir la surveillance effective et celle d'une organisation disciplinée de sa leçon. Investiguer la dynamique des échanges langagiers dans ce contexte n'est donc pas sans intérêt.

Les choix méthodologiques pour appréhender ce qui se passe « d'ordinaire dans les situations didactiques " s'inscrivent dans l'approche clinique/expérimentale (Leutenegger, 2000, 2009 ; Schubauer-Leoni \& Leutenegger, 2002). Cette dernière permet d'étudier le fonctionnement du système didactique en tâchant d'articuler des informations recueillies pendant les séances d'enseignement/apprentissage avec celles recueillies pendant des entretiens. Le dispositif utilisé articule trois types de recueil de données auprès de deux enseignants et deux élèves.

L'entretien ante cible le décours temporel de la leçon. II explore les conceptions annoncées de l'enseignant sur l'activité, sur l'enseignement, sur l'apprentissage pour repérer d'éventuelles récurrences dans les comportements, les gestes et les énoncés qui pourraient y être imputables. Il permet de repérer des facteurs de permanences qui pourraient renvoyer à des compétences stabilisées, à des schèmes (Vergnaud, 2007). II interroge les conceptions de l'enseignant sur la notion de contenu (de la conception à l'éventuelle formulation aux élèves) pour situer le niveau de transposition didactique où l'enseignant élabore ses consignes verbales et pour identifier le rapport entretenu entre consignes verbales et ce qu'il y a à apprendre. Enfin, il permet d'appréhender les conceptions de l'enseignant sur la dynamique de la transmission de ces contenus (gestes professionnels anticipés et prévus): l'enseignant accorde-t-il une place à sa gestuelle, à son activité langagière, au contexte de production de sa communication ? Est-ce un champ sur lequel porte sa réflexion professionnelle?

La prise de vidéo de l'enseignant en cours d'action en plan large avec enregistrement audio synchronisé (dictaphone embarqué) permet de recueillir des traces archivables du cours d'action : cette observation correspond aux activités produites par l'enseignant et les élèves «constitutives de leur travail respectif, co-production de phénomène topo, chrono et mésogénétique » (Schubauer-Leoni \& Leutenegger, 2002). Elle est associée aux préparations de leçon remises sous forme écrite.

Enfin, l'entretien post vise à recueillir «l'avis, le sentiment, l'analyse réflexive du professionnel (et des élèves) après l'accomplissement d'une séance de travail » (Schaubeuer-Leoni \& Leutenegger, 2002). Nous avons opté pour un entretien d'autoconfrontation (Theureau, 2010) et d'autoconfrontation croisée afin de saisir le sens des 


\section{eJRIEPS 42 juillet 2017}

écarts constatés par rapport aux prescriptions (Theureau \& Jeffroy, ibid 1994 ; Leplat, 2000). Nous avons procédé en trois phases, à partir de séquences vidéo sélectionnées. Le protocole s'initie par une autoconfrontation visant à mettre en explicitation l'enseignant sur les déterminants situationnels qui ont présidé aux choix, à l'émergence, à la priorisation des gestes professionnels, leur répartition et leur distribution (verbal/coverbal/non-verbal). II s'agit d'installer l'enseignant en situation de conscience préreflexive, sans déborder sur la réflexion consciente (Theureau, 2010). Ensuite, une autoconfrontation avec deux élèves relevant de niveaux de pratique différents a permis d'identifier la charge sémique coconstruite enseignant/élève. Enfin, une autoconfrontation croisée avec un second enseignant, visant à identifier le degré de savoir professionnel commun en jeu dans les alternances de canaux de communication complète le dispositif.

Deux enseignants ont accueilli le projet de recherche favorablement. Le corpus de données est constitué à partir de leurs deux classes de terminales, de profil comparable, avec une échéance d'évaluation certificative permettant de limiter les biais des niveaux de développement et de la motivation. Deux élèves par classe et de niveaux différents déterminés par l'enseignant se sont prêtés aux entretiens post séance. Au total, nous disposons de six séquences vidéo (+audio) d'une durée totale de 150 minutes (leçon d'EPS), de séquences audio sur dictaphone d'une durée totale de 45 minutes (entretiens) ainsi que de quatre séquences vidéo/audio d'autoconfrontation d'une durée totale de 72 minutes.

Le traitement des données a été réalisé en trois phases. Premièrement, les entretiens ont fait l'objet d'une analyse de contenu (Bardin, 1991) selon trois règles d'énumération: la présence/absence, la fréquence et la co-occurence (niveau/démarche d'intervention). Nous avons identifié six catégories : le contenu technique normé, le contenu action/compréhension, la démarche pédagogique, l'organisation spatiotemporelle, le niveau des élèves et la conscientisation de la gestuelle.

Deuxièmement, pour les séquences vidéos, nous avons procédé à des visionnages successifs portant sur les actions de l'enseignant dont le découpage et le dénombrement s'est fait en fonction des silences, des déplacements, des changements d'interlocuteurs. Cette lecture a été guidée par cinq critères : (1) les actions chronologiques, (2) le temps consacré à chaque action, (3) la direction dans l'enchainement des actions, (4) l'association de chaque action à un niveau de pratique de l'élève (ou groupe d'élèves concerné : couleur de bonnet identique par niveau), (5) le redécoupage des actions didactiques (définition, dévolution, régulation, institutionnalisation). Nous avons séparé les relevés des deux enseignants. 


\section{eJRIEPS 42 juillet 2017}

Ensuite, nous avons procédé à un relevé des occurrences totales (L1 à L3) et occurrences partielles (L1) (L2) (L3), sur les registres coverbal / verbal / non verbal / silence (gestuel et verbal) chez chacun des deux enseignants, de façon différenciée puis fusionnée. Toutes les données ont été ramenées à des valeurs en pourcentage. Le second axe de relevés concerne les temps totaux et partiels sur les différents registres, à partir desquels nous avons retenu une durée moyenne en divisant les temps par le nombre d'occurrences correspondants. Puis, nous avons procédé à des relevés sur la direction des changements de registre d'intervention c'est-à-dire la direction des transitions (du verbal vers le gestuel par exemple) sur L1-L2-L3 et L1-L3 pour les répertorier afin ensuite d'en effectuer un dénombrement ramené en pourcentage.

Enfin, nous avons réalisé une série de tris croisés au regard de deux variables : les actions didactiques et le niveau dans l'APSA dont dépend « la capacité à lire et interpréter les signes émis par l'enseignant» (Mahut, 2003, p. 101). Pour chacun d'eux, nous avons relevé les pourcentages d'occurrences par enseignant et par leçon. Ensuite, ont été calculés les temps des registres de communication puis explorée la direction des transitions.

\section{Résultats et discussion}

5. 1. Modélisation des transitions codiques

L'analyse des enregistrements vidéo fait apparaitre que le canal de communication majoritairement emprunté par les enseignants est verbal, avec $37,04 \%$ des occurrences d'interactions communicationnelles produites, contre $28,68 \%$ pour la gestuelle coverbale, $16,22 \%$ pour les silences (verbaux et gestuels), 17,21\% pour les déplacements. La gestuelle brute est quasiment absente (0,49\%) (Graphique 1). Ainsi est mise en évidence une hiérarchie dans l'usage des registres de communication: le verbal / le coverbal / le silence et les déplacements. Ces données sont confortées par celles relatives d'une part au temps consacré à chacun des canaux de communication, et d'autre part à l'analyse comparée des données sur les deux enseignants en occurrences, durée et direction. 


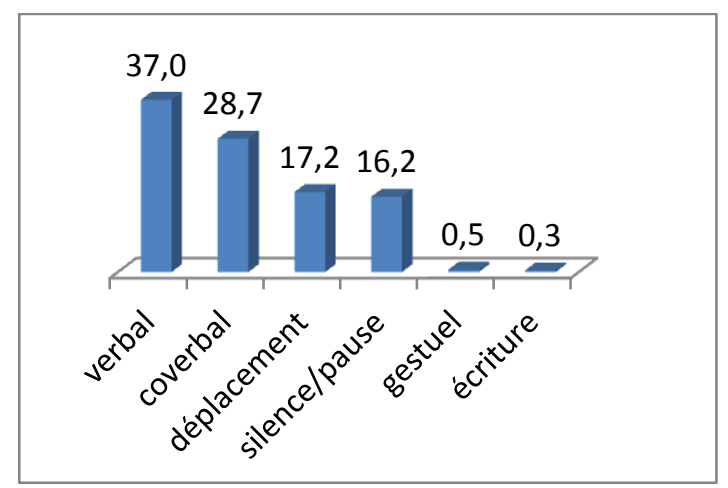

Graphique 1. Répartition des communications selon les registres.

Ces résultats restent inchangés lorsque l'on procède à une analyse par situation d'apprentissage. Les objets de savoir mis à l'étude ne semblent pas impacter cette dynamique. En revanche, les résultats contredisent les énoncés des entretiens ante au cours desquels les enseignants indiquent associer la transition codique à une volonté de mieux se faire comprendre par les élèves et ce, selon une organisation temporelle repérée du type canal verbal, puis canal gestuel, puis canal coverbal, à l'instar de ce professeur qui déclare : " Je démarre toujours avec une définition verbale et ensuite j'utilise presque à chaque fois les gestes et puis quand cela ne va pas j'utilise les deux en même temps...». Trois conclusions s'ébauchent alors : d'abord, il existe une épistémologie pratique sous-jacente chez les enseignants relative aux transitions codiques; ensuite, la mise en œuvre de cette conception de l'intervention ne semble pas aller de soi et se trouve contrariée par l'interaction didactique; enfin, à en juger par les fiches de séances associées à l'entretien ante, les enseignants, dans la phase de construction des tâches, ne portent pas leur réflexion professionnelle sur la façon de transmettre les contenus, mais essentiellement sur l'élaboration de ces derniers. Ces données sont convergentes avec les travaux déjà réalisés dans le domaine (Boisumault \& Cogerino, 2012; Brown, 2000). Les actions de communications et leur enchainement réalisés en classe sont « non calculés », comme le souligne le premier enseignant: “Ce n'est pas calculé et...c'est en prise avec les informations sur le tas, ce n'est pas réfléchi du tout »; et l'alternance reste inféodée à des connaissances théoriques d'arrière-plan qui sont convoquées pour guider les interventions, comme par exemple le niveau scolaire des élèves et leur profil pédagogique, auxquels est associé un type de contenu d'enseignement, variables envisagées comme déterminantes et concurrentes dans les enchainements codiques. Ainsi, un enseignant précise : « Le contenu à faire passer avec les élèves scientifiques (....)vu le niveau en physique et en math c'est la compréhension de l'action à faire mais aussi le concept qu'il y a derrière, d'un point de vue 


\section{eJRIEPS 42 juillet 2017}

biomécanique par exemple [...] Je pense que je dois être verbal au début, et là ce ne sont pas des débutants donc je vais être très proche sur la démonstration, je vais utiliser un élève qui maitrise ou soit permettre aux autres de réguler avec son vocabulaire [...] Parce que j'imagine que l'élève peut comprendre de différentes manières et que je ne peux savoir à l'avance ce qui va le toucher plus... donc j'essaie d'avoir un panel heu... d'actions pour que l'élève soit touché par l'une ou l'autre ". On voit bien, dans cet énoncé à quel point se combinent épistémologie pratique, construction et élaboration des tâches mises en étude, et genèse des transitions codiques en interaction. Les relevés effectués sur les trois leçons d'apprentissage auprès des deux enseignants montrent que plus du tiers des directions engagées articulent le verbal au coverbal (23,48\% et $12,32 \%)$. Ces données nous permettent également observer que près d'un tiers des changements de mode de communication ont lieu en étant associés soit à des silences, soit à des déplacements de l'enseignant.

Ces deux critères, constituants souvent des balises temporelles de l'intervention, sont donc pertinents pour notre étude (graphique 2).

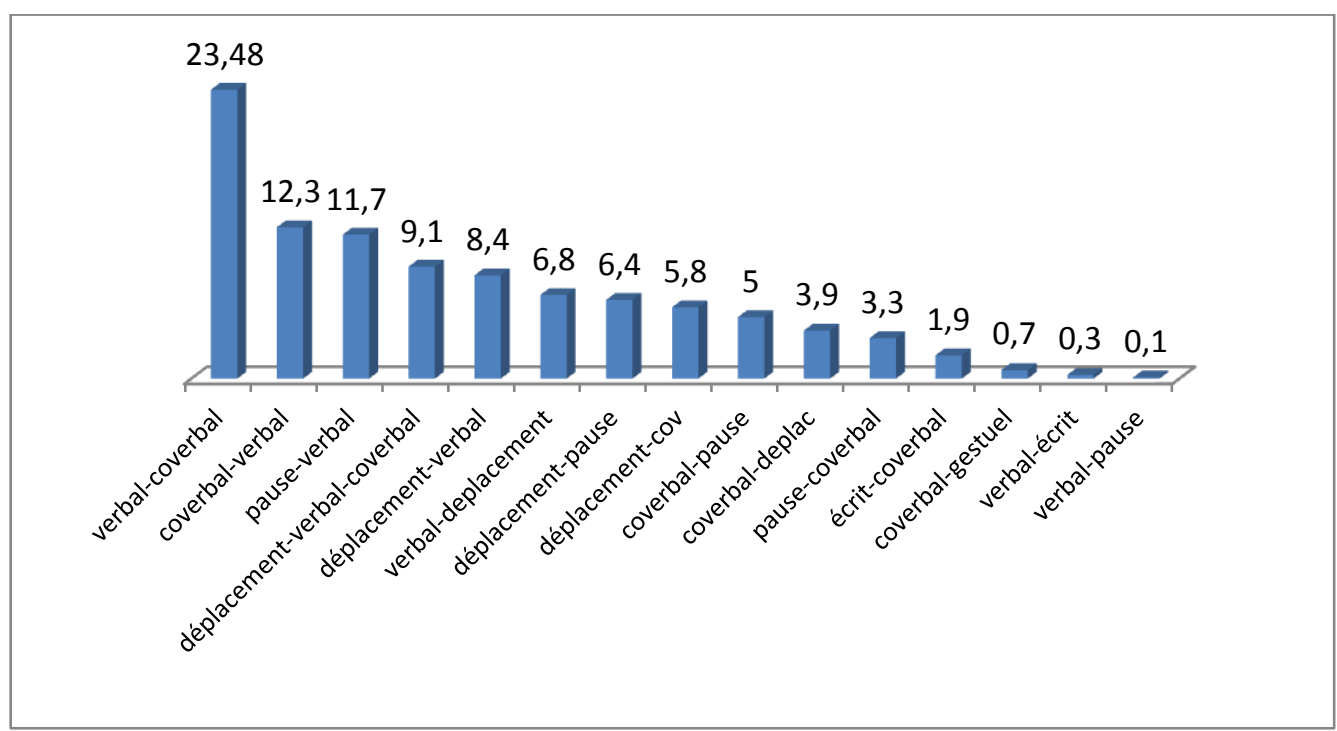

Graphique 2. Occurrences directionnelles des deux enseignants (résultats en pourcentage).

La distribution ainsi que la dynamique des transitions codiques se différencient également en fonction du type d'action didactique engagée, que ce soit du point de vue des occurrences, de la temporalité et de la direction. La comparaison des deux enseignants au niveau mésodidactique (graphiques 3 à 6), nous permet de relever des similitudes de fonctionnement : un équilibre temporel entre interventions verbales et coverbales dans les actions de régulation, 


\section{eJRIEPS 42 juillet 2017}

et plus de temps accordé aux interventions coverbales dans celles de définition. Les variations constatées sont interprétées par les enseignants au regard de la démarche différenciée annoncée en entretien ante : directive et transmissive pour E1 et incitative et par l'action pour E2. La transition verbal-coverbal est très utilisée en phase de définition $(30,33 \%)$ et d'institutionnalisation $(27,2 \%)$, mais beaucoup moins au moment de la dévolution $(17,9 \%)$. Au cours de la régulation, ce sont les transitions pause-verbal qui sont les plus empruntées, comme si l'activité d'observation précédant l'intervention, à ce moment des interactions, devenait un schème caractéristique de l'agir enseignant. Si on dissocie, dans la gestuelle coverbale, les éléments spatiographiques et les éléments kinétographiques, nous obtenons une distribution des occurrences et temporalités, sur les moments didactiques, différenciée et évolutive d'une leçon à l'autre (graphique 7 à 12). C'est dans les actions de définition et de régulation que les occurrences kinétographiques sont les plus importantes, et en phase de définition et de dévolution que les occurrences spatiographiques le deviennent.

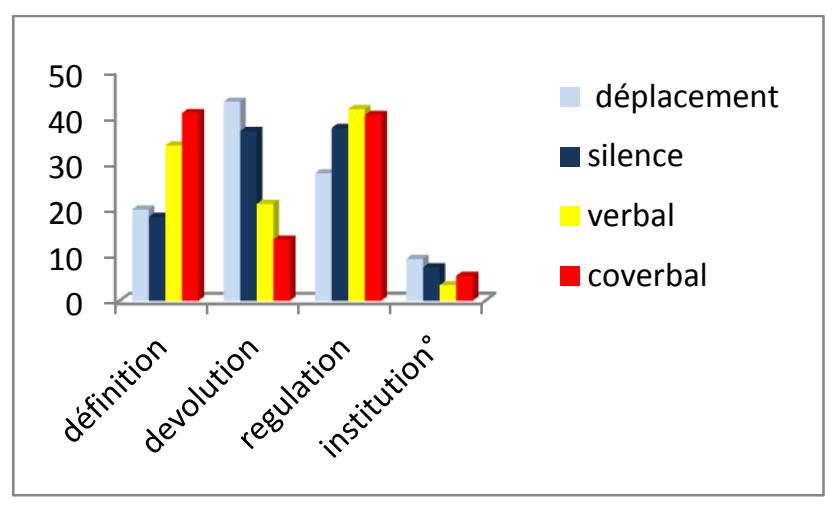

Graphique 3. Occurrences selon les moments didactiques enseignant $1(\mathrm{E} 1)$.

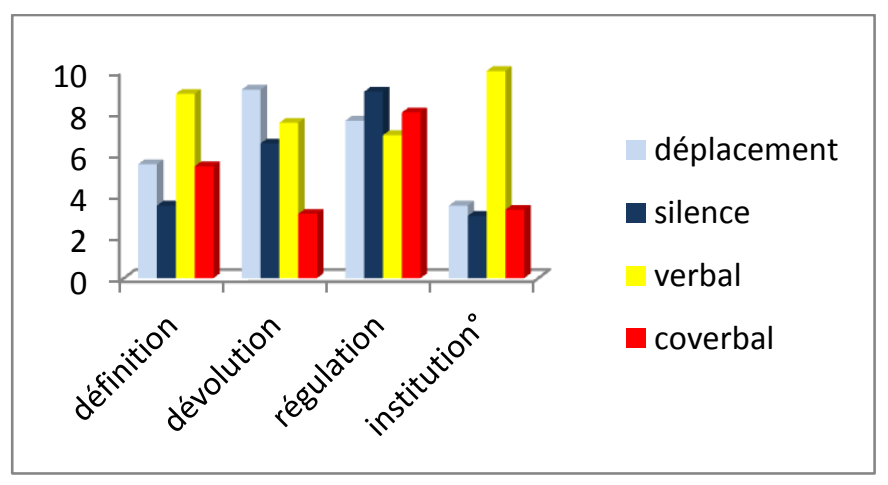

Graphique 5. Temporalité selon les moments didactique $\mathrm{E} 1$.

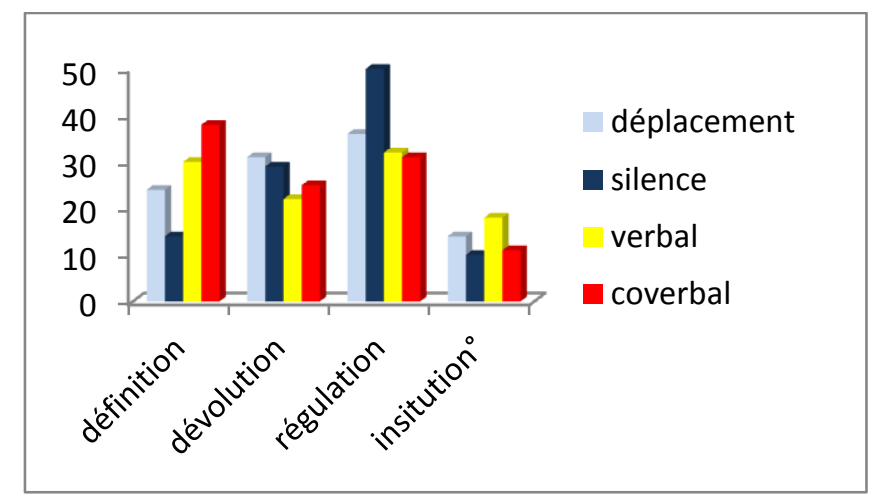

Graphique 4. Occurrences selon les moments didactiques enseignant 2 (E2).

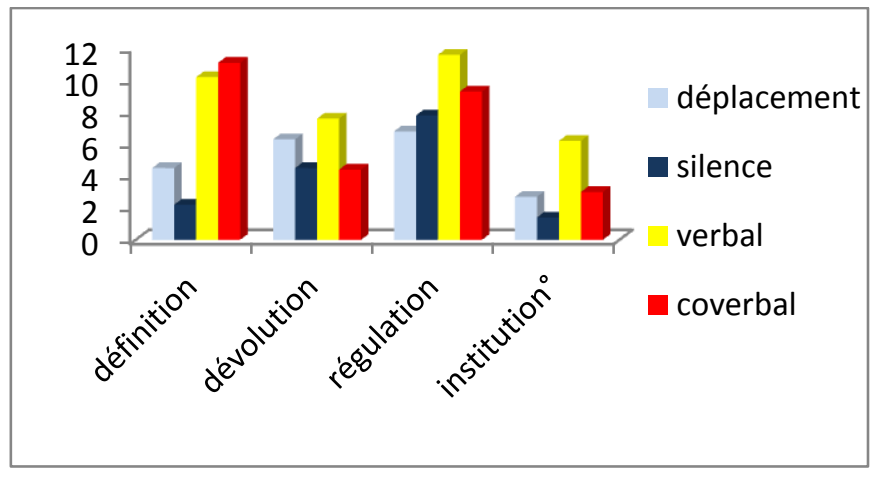

Graphique 6. Temporalité selon les moments didactiques E2. 


\section{eJRIEPS 42 juillet 2017}

Sur la dynamique évolutive des répartitions, la transformation dans l'utilisation de l'une ou l'autre des dimensions coverbales la plus flagrante se situe au niveau des moments de régulation didactique où l'on passe d'une dominante spatiographique en leçon 1 chez l'enseignant 1 à une exclusivité de l'exploitation de la dominante kinétographique en leçon 3. Les stratégies d'intervention coverbales évoluent donc d'une leçon à l'autre, mais ne touchent pas de façon équivalente les actions didactiques. En phase de définition, on voit l'augmentation du registre spatiographique, ce qui signifie que le recours à la démonstration s'efface au profit de gestes explicatifs sans doute plus directs, plus courts, mais aussi plus codés en interaction avec les élèves. Chez l'enseignant 2, on constate de la stabilité entre le kinétographique et le spatiographique, avec une égalité permanente d'une leçon à l'autre entre les gestes spatiographiques dans les actions de dévolution et les actions de régulation.



Graphique 7. Cov selon le moment didactique L1 (E1).



Graphique 10. Cov selon le moment didactique L1 (E2).



Graphique 8. Cov selon le moment didactique L2 (E1).



Graphique 11. Cov selon le moment didactique L2 (E2).

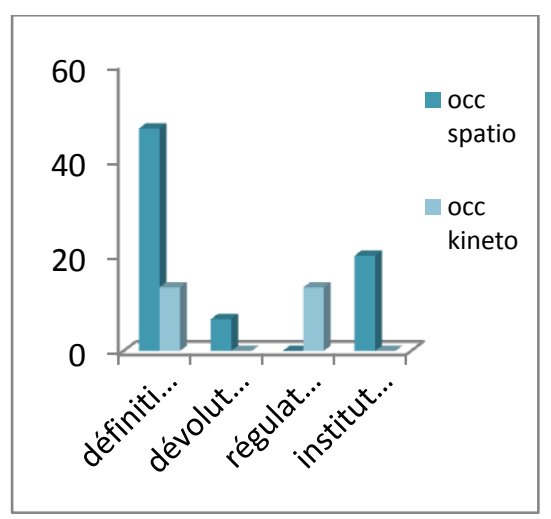

Graphique 9. Cov selon le moment didactique L3 (E1).

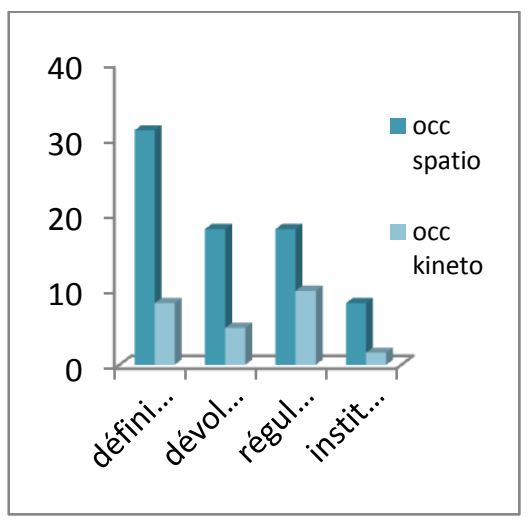

Graphique 12. Cov selon le moment didactique L3 (E2).

Légende: pour « defi », lire définition, pour « dév», lire dévolution, pour « régu », lire régulation, et pour « insti », lire institutionnalisation. 


\section{eJRIEPS 42 juillet 2017}

On peut s'interroger sur la relation à établir entre cette stabilité (chez E1) et l'annonce faite d'une certaine directivité dans les entretiens ante. Le graphique 13 met en évidence que la distribution des registres de communication ne s'effectue pas simplement en fonction des niveaux. On constate effectivement plus d'interventions coverbales que verbales au niveau 3 , un équilibre au niveau 2 et plus d'interventions verbales que coverbales au niveau 1. Cependant, les occurrences et les temporalités, qui se correspondent, décroissent au fur et à mesure que le niveau des élèves augmente comme s'il s'agissait d'intervenir plus et plus souvent au niveau 1. Nous noterons que c'est à l'occasion des interventions coverbales que les écarts entre les niveaux sont les moins importants.

Les transitions codiques sont donc modélisables à partir des trois critères que nous avons retenus : occurrences, temporalité et directions. Cette caractérisation s'affine en fonction des actions didactiques mais aussi en fonction des niveaux de pratique des élèves. Cette différenciation des transitions codiques au regard des différents niveaux de maitrise des élèves dans la classe semble caractériser l'émergence et la gestion qualitative (par les registres de communication) et quantitative (occurrences et temporalités) par l'enseignant de contrats didactiques différentiels. Cette analyse méso-didactique rendant compte des phénomènes transitionnels codiques doit donc être complétée par une analyse microdidactique.



Graphique 13. Distribution des occurrences selon le niveau des élèves. 


\section{eJRIEPS 42 juillet 2017}

5. 2. La production des contenus : point d'équilibre entre savoirs académiques, savoirs professionnels et effets de contextes liés à la gestion professorale des contraintes.

\section{2. 1. Analyse micro-didactique des transitions codiques}

Cette analyse va reposer sur des extraits représentatifs des interactions entre les enseignants et leurs élèves.

\begin{tabular}{|c|c|c|}
\hline Ce que dit l'enseignant & Ce que fait l'enseignant & Chronologie \\
\hline $\begin{array}{l}\text { Quand vous faites le virage } \\
\text { culbute, je vous rappelle que } \\
\text { quand vous êtes à une longueur } \\
\text { de bras du bord, c'est le } \\
\text { moment de basculer la tête en } \\
\text { avant, à cette distance là ; } \\
\text { trop proche, vous allez toucher } \\
\text { le mur quand vous allez } \\
\text { basculer surtout avec vos pieds } \\
\text { soit vous êtes trop loin et vous } \\
\text { ne toucherez pas le mur } \\
\text { donc ça c'est la bonne distance }\end{array}$ & $\begin{array}{l}\text { Statique et droit, explique } \\
\text { Allonge le bras devant } \\
\text { Déplacement vers le poteau } \\
\text { Allonge le bras devant le poteau } \\
\text { Bascule la tête } \\
\text { Remet le bras pour réindiquer la } \\
\text { bonne distance } \\
\text { Se rapproche du poteau et } \\
\text { mime le virage } \\
\text { S'éloigne du poteau } \\
\text { Remontre la bonne distance }\end{array}$ & $\begin{array}{l}0^{\prime}----5^{\prime \prime} \\
5^{\prime}---7^{\prime \prime} \\
7^{\prime \prime}---10^{\prime \prime} \\
10---12^{\prime \prime} \\
12^{\prime \prime}-13^{\prime \prime} \\
13^{\prime \prime}-14^{\prime \prime} \\
14^{\prime \prime}---20^{\prime \prime} \\
20^{\prime \prime}---23^{\prime \prime} \\
23^{\prime \prime}-24^{\prime \prime} \\
24^{\prime \prime}-25^{\prime \prime}\end{array}$ \\
\hline & & Les élèves agissent \\
\hline
\end{tabular}

Dans cet extrait, nous sommes dans une phase, en début de leçon, où l'enseignant pose la définition de la tâche relative au savoir mis à l'étude : le virage. Tous les élèves sont en écoute mais les consignes sont adressées au groupe niveau 3. Nous constatons d'abord qu'après une phase initiale courte de communication verbale, l'enseignant initie une série de gestes accompagnant la parole (coverbale), chacun ayant pour fonction d'expliciter les attendus. L'importance accordée à cette communication coverbale est conforme à l'ensemble des relevés en phase de définition. On constate que les enchainements d'actions sont relativement courts et que la densité ainsi que la redondance des 


\section{eJRIEPS 42 juillet 2017}

informations enrichissent le milieu didactique : la mésogénèse se développe donc au regard des transitions codiques impulsées. Par ailleurs, on remarque également que le savoir mis à l'étude initialement se spécifie au fur et à mesure de l'intervention de l'enseignant, pour finalement se focaliser sur une phase précise du virage culbute: le moment de son déclenchement. Le contrat didactique est organisé pour l'enseignant autour de ce moment de déclenchement, pour l'élève autour d'une production de rotation à partir d'une bonne distance de déclenchement. L'enseignant explique en démontrant, de façon précise et redondante, ce qu'il convient de faire pour obtenir la bonne distance de déclenchement, sans pour autant donner d'indication sur la rotation elle-même, laissée en dévolution aux élèves. Les contenus sont de nature informationnelle basés sur des repères pouvant être pris en compte par les élèves (visuels et spatiaux). La chronogénèse est donc déclenchée par ce type de contenus mais également par des contenus représentationnels permis par l'étalonnage de la bonne distance cernée entre deux distances inadéquates (trop près et trop loin par rapport à la distance de bras). La convocation de la mémoire didactique active cette chronogenèse (« je vous rappelle... »). A cet instant, la topogenèse est plutôt en accompagnement puisque l'essentiel de l'intervention vise à mettre en réussite les élèves sans leur donner les solutions techniques au virage lui-même.

\begin{tabular}{|c|c|c|}
\hline Ce que dit l'enseignant & Ce que fait l'enseignant & Chronologie \\
\hline $\begin{array}{l}\text { ceux qui ne font pas la, la heu le } \\
\text { virage culbute, vous allez faire } \\
\text { le virage demi tour } \\
\text { quand j'arrive je me retourne le } \\
\text { plus rapidement possible, je me } \\
\text { regroupe pour aller vite, on } \\
\text { reprend la position allongée et } \\
\text { vous repartez et quand vous } \\
\text { arrivez de l'autre coté vous } \\
\text { enchaînez } \\
\text { d'accord?, je vais aller de } \\
\text { l'autre coté pour regarder } \\
\text { comment vous vous y prenez et } \\
\text { vous faites un bilan. }\end{array}$ & $\begin{array}{l}\text { Se déplace } \\
\text { Explique } \\
\text { Mime la rotation } \\
\text { Explique } \\
\text { Mime le regroupement } \\
\text { Explique } \\
\text { Montre la direction } \\
\text { explique } \\
\text { montre la direction et tourne le } \\
\text { poignet } \\
\text { se déplace }\end{array}$ & $\begin{array}{l}25--26 \\
26---34 \\
34---35 \\
35-37 \\
37-38 \\
38-42 \\
42-43 \\
43-49 \\
49-53 \\
53--55 \\
55-1^{\prime} 07\end{array}$ \\
\hline & & Les élèves agissent \\
\hline
\end{tabular}




\section{eJRIEPS 42 juillet 2017}

Dans cet extrait qui fait immédiatement suite au précédent, l'enseignant s'adresse à des élèves jugés de niveau moins efficace (niveau 1) que les précédents dans les nages, en proposant, dès ses actions de définition, une mise en étude du virage différente : le virage demi-tour. On remarquera que les transitions codiques s'organisent différemment que pour le groupe de niveau 3 puisque nous avons une alternance de communications verbale et co-verbale qui s'équilibrent au service d'explications démontrées, la part de verbalisation étant supérieure à la phase de coverbalisation, ce qui illustre au plan microdidactique les relevés effectués sur l'échelle méso-didactique. Le contrat didactique pour l'élève est ici organisé autour de la construction d'une « bonne » représentation du virage, dans toute sa chronologie. Pour l'enseignant, le contrat s'appuie sur les attendus à obtenir, et dans la perspective conjointe, les ajustements vont s'organiser autour d'une forme gestuelle à produire. Du point de vue des contenus, les repères donnés aux élèves s'évaporent en démonstration des différentes phases sans qu'il y ait, comme pour le groupe précédent, une focalisation sur une phase précise pouvant permettre aux élèves des avancées décisives dans leur apprentissage. On remarquera ainsi que la chronogenèse est produite par cette alternance de démonstrations explicitées et explications, avec une énonciation du comportement terminal de réponse. La mésogenése ne s'épaissit pas dans la mesure où aucune action ou information n'est donnée à l'élève pour qu'il interagisse autrement avec le milieu. La topogenèse est ici en surplomb puisque l'on donne la solution attendue, et en même temps, la réticence didactique est absente. L'enseignant ne prend pas en compte le déjà là des élèves et ne propose pas d'élément à prendre en compte pour organiser leur transformation. On remarquera également qu'entre les deux niveaux d'élèves, le délai de mise en activité des élèves est allongé pour le niveau 1, ce qui écourte leur temps de pratique motrice et, à terme, hypothèque leur apprentissage. Ce phénomène est accentué si l'on considère que le premier extrait a profité à tous (N1 et N3) en étant pourtant adressé au niveau 3, et que le suivant est adressé au niveau 1, sans qu'aucun trilogue groupal puisse être envisagé. C'est donc que le groupe 1 subit une charge informationnelle inadéquate en même temps qu'un temps de pratique plus réduit.

Cette analyse micro-didactique rend compte d'une dynamique différentielle du doublet contrat-milieu didactique : lorsque l'enseignant procède par communication coverbale, la dynamique des contenus s'organise autour de repères sensorimoteurs et spatiotemporels qui, a priori, sont compatibles avec l'efficacité dans la tâche proposée et garantissent dès lors l'appropriation du savoir mis en étude. Ces repères permettent à l'élève d'agir 


\section{eJRIEPS 42 juillet 2017}

différemment dans le milieu proposé, mais également de mieux prendre en compte ce que le milieu lui renvoie comme informations. Par ailleurs, lorsque l'enseignant procède davantage avec une communication verbale, la dynamique des contenus se spécifie autour de règles d'actions ou de chronologies d'actions à respecter. Leur degré de généricité et leur caractère formel semblent ne pas permettre aux élèves d'agir différemment ou de prendre en compte d'autres rétroactions du milieu. Or, les constats révèlent que la dimension verbale est exploitée prioritairement pour les élèves de niveau 1 et la dimension coverbale l'est pour les élèves de niveau 3. Cette perspective semble être à la base d'un mécanisme construisant/renforçant les inégalités au sein d'une même classe. De plus, les entretiens réalisés avec les élèves laissent anticiper certaines conséquences accentuant cette tendance : ils révèlent en effet que les lycéens repérés à un niveau modeste de pratique demandent que soit privilégiée «la démonstration concrète du geste [...] et ce plusieurs fois ». Ils ne sont donc pas en situation de communication favorable pour apprendre de part cet effet de contexte sociocognitif généré par une modalité de communication que l'élève n'attend pas. En revanche, les élèves repérés comme ayant acquis un niveau plus élaboré semblent indifférents aux canaux de communication, même s'ils manifestent une préoccupation de conformisme aux demandes de l'enseignant. On comprend alors que dans cette attente différentielle, la question de l'orchestration des différents canaux de communication pour satisfaire chacun reste une compétence à construire.

5. 2. 2. Analyse méso-didactique de la dynamique des transitions codiques Le retour à une échelle d'analyse plus étendue permet de pondérer les résultats antérieurs.L'étude de la dynamique de l'articulation des canaux de communication durant les trois leçons successives chez les deux enseignants indique une évolution contrastée des occurrences verbales et coverbales fonctionnant en compensation inverse. Au déséquilibre initial $(\mathrm{V}>\mathrm{COV})$ succède un équilibre codique $(\mathrm{V}=\mathrm{COV})$. 


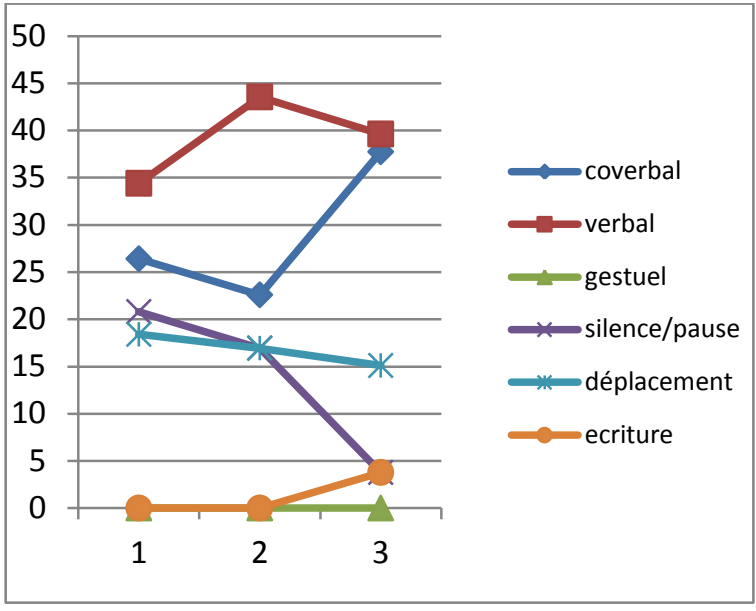

Graphique 14. Evolution des occurrences pour E1 selon le numéro de la leçon (en abscisse).

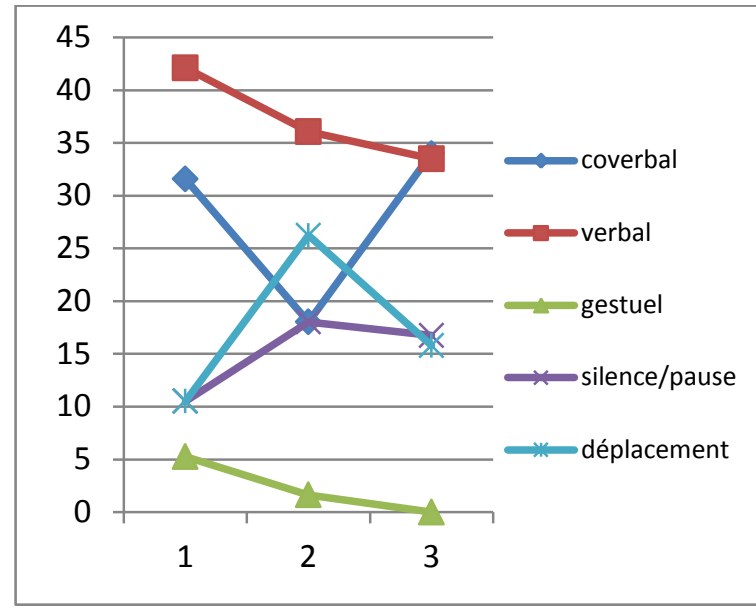

Graphique 15. Evolution des occurrences pour E2 selon le numéro de la leçon (en abscisse).

Ces données sont en décalage avec ce que les enseignants annoncent en entretien ante, confirmant à la fois l'imperméabilité à la conscience des transitions codiques dans l'interaction et le décalage entre l'épistémologie pratique sous-jacente et l'intervention. Les entretiens d'autoconfrontation permettent d'obtenir des explications sur ces distorsions. Les enseignants les attribuent aux contraintes qui pèsent sur l'enchainement des canaux de communication dans l'interaction comme :

- le niveau des élèves, “je pense que je dois être verbal au début et là ce ne sont pas des débutants donc je vais être très proche sur la démonstration "(E2)

- le temps d'intervention, «il faut absolument que les élèves soient en action, ...action, action, action.... surtout sur une séance de natation qui est de 50 à 55 minutes, c'est très limité ; c'est une contrainte énorme »(E2)

- et le contexte d'intervention "j'essaie de me mettre exactement comme elle (une élève) va être dans l'eau donc je me rapproche..., là j'interviens en appelant l'élève mais c'est difficile en natation pour corriger quelque chose qui n'allait pas au milieu du bassin... »(E1).

La comparaison des données chiffrées et du contenu des entretiens chez les deux enseignants laisse penser que le choix de leur démarche et la nature des contenus ciblés pour les leçons auraient un impact sur les transitions codiques. Cependant, on constate une évolution importante dans l'exploitation des canaux de communication qui se localise au moment des phases de régulation. Au cours des enseignements, les articulations entre canaux de communication évoluent donc de façon non linéaire et traduisent finalement le résultat de ce qui se joue au moment de l'intervention de l'enseignant, trahissant des 


\section{eJRIEPS 42 juillet 2017}

contradictions entre une démarche avouée et repérable et les nécessités de faire avancer le savoir face à des élèves qui manifestent des réponses pas forcément en adéquation avec les attentes de l'enseignant. Ainsi on trouvera un enseignant directif (E1) mais qui utilise de plus en plus de silences en phase de régulation et moins de verbes en définition, et un enseignant soucieux de parler le moins possible (E2) mais qui utilise de plus en plus le verbe au moment des régulations.

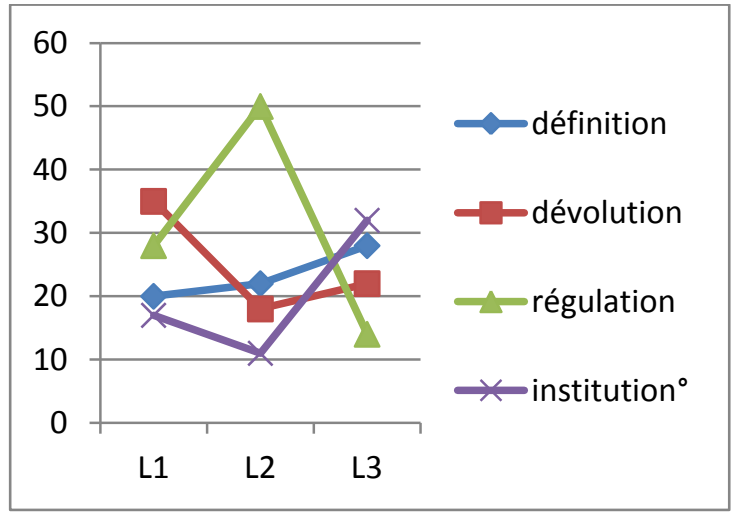

Graphique 16. Evolution des occurrences des verbes en fonction du moment didactique (en \%) chez E1.



Graphique 18. Evolution des occurrences des verbes en fonction des moments didactiques (en \%) chez E2.

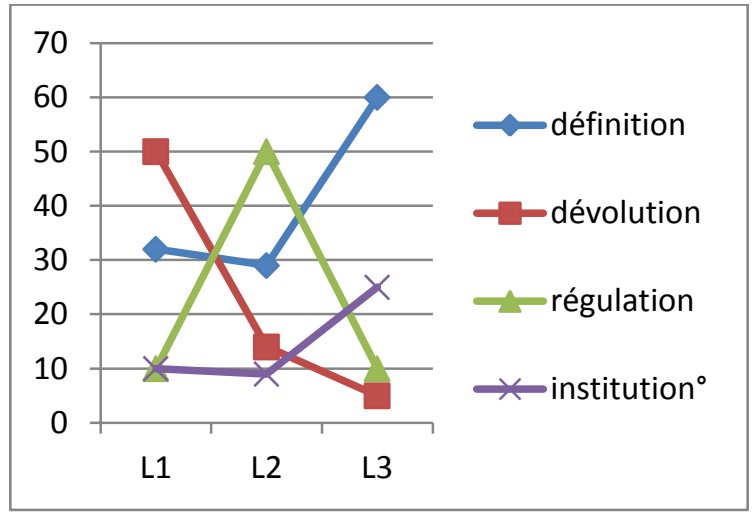

Graphique 17. Evolution des occurrences de la gestuelle coverbale en fonction du moment didactique (en \%) chez E1.



Graphique 19. Evolution des occurrences des gestuelles coverbales en fonction des moments didactiaues (en \%) chez E2.

Cet ensemble de résultats, associés aux données d'entretien, indiquent que la dynamique des transitions codiques émerge d'un système de contraintes qui s'imposent à l'enseignant alors que celui-ci réalise des choix intuitifs de modes de communication. Suite à ces choix, des effets de contextes sociocognitifs (décalage entre les attendus des élèves en termes de 


\section{eJRIEPS 42 juillet 2017}

canal communicationnel et les attendus de l'enseignant en termes de réponses motrices) vont émerger et contraindre en retour l'enseignant à adapter sa démarche et son mode de transmission en situation, contrariant les fondements de son épistémologie pratique en ce domaine.

II apparait également que la dynamique des interventions s'organise dans le sens d'une diminution des interventions verbales et coverbales en fonction des niveaux de pratique. Pour le niveau 3 par exemple, on constatera que les temps de communications coverbales, comme les occurrences, diminuent entre L1 et L3 chez les deux enseignants. (graphique 20 à 25) mais augmentent pour le niveau 1.

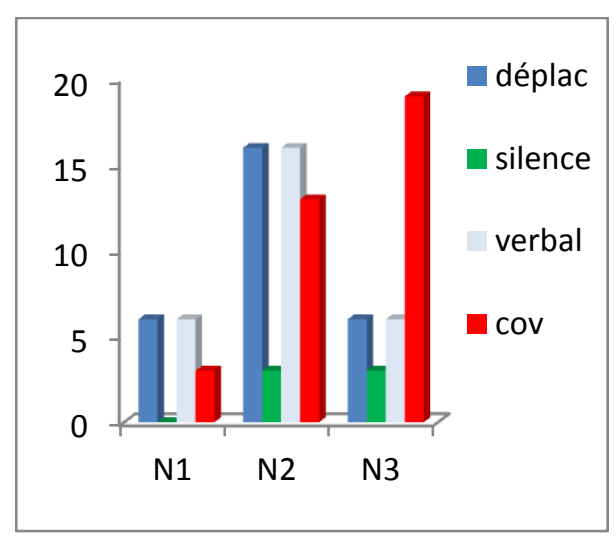

Graphique 20. Occurrences leçon 1 par niveau (E1).



Graphique 23. Occurrences leçon 1 par niveau (E2).

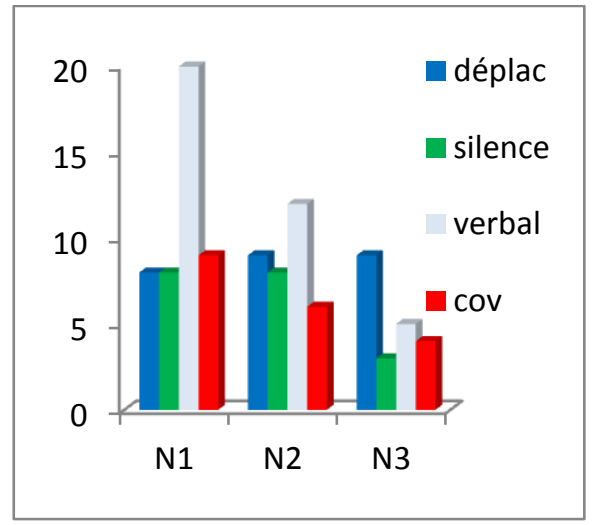

Graphique 21. Occurrences leçon 2 par niveau (E1).



Graphique 24. Occurrences leçon 2 par niveau (E2).

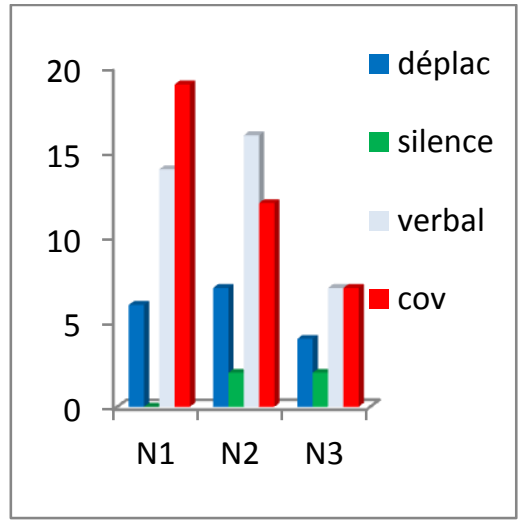

Graphique 22. Occurrences leçon 3 par niveau (E1).

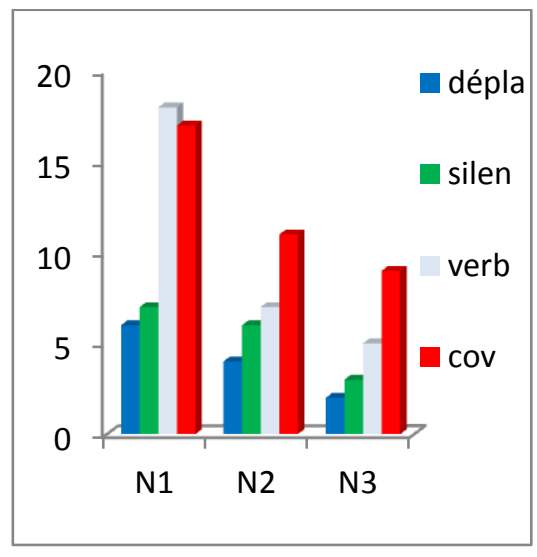

Graphique 25. Occurrences leçon 3 par niveau (E2).

Légende : pour « déplac » lire déplacement, pour « cov » lire coverbal

Ces résultats viennent donc porter un éclairage nouveau sur l'analyse microdidactique réalisée plus tôt caractérisant un contrat-milieu didactique différentiel. II semblerait que les interventions coverbales relèvent d'une charge sémique progressivement accessible à des 


\section{eJRIEPS 42 juillet 2017}

élèves ayant moins de prérequis moteurs et directement accessible à ceux qui ont un capital important de vécu dans l'activité physique et sportive sélectionnée. Autrement dit, l'exploitation du canal coverbal impose un recours au sens qui doit se construire à travers l'interaction enseignant/élève et dont le décodage dépend du niveau du vécu moteur dans l'APSA considérée. Cette interprétation interroge alors le curriculum enseigné par la façon de l'enseigner : il devient nécessaire pour l'enseignant d'adapter ses canaux de communication selon les différents profils d'élèves de sa classe, sous peine de les empêcher d'apprendre. C'est ce que montrent nos données chiffrées croisées avec les entretiens.

Les transitions codiques sont une réponse liée à la fois à une réaction de l'enseignant en situation et à des contraintes institutionnelles ou des savoirs professionnels (éthique de l'équité ou profils pédagogiques). Nous conviendrons de conclure que la stratégie d'intervention initiale de l'enseignant en fonction des groupes d'élèves dans la classe se modifie dans l'interaction et au fil de la chronogénèse. Les logiques d'arrière-plan cèdent donc la place à des coconstructions codiques dans les échanges. Au départ, la dynamique des registres de communication est liée à différentes contraintes : celles des savoirs sur les élèves (niveau de maîtrise), celles des savoirs sur le maintien d'un climat communicationnel (directivité initiale chez les deux enseignants), celles des savoirs sur l'apprentissage (profils cognitifs), celles liées aux contextes physiques (éloignements enseignant-élève, hauteurs et similitudes des positions). Ensuite cette dynamique évolue sous l'effet d'une association faite par l'enseignant entre ses types d'interventions et leurs effets inattendus sur la motricité des élèves, phénomène interprété alors comme un manque de compréhension lié au niveau de réception de l'élève ou au manque d'information initiale (charge sémique faible). Notre seconde hypothèse semble donc ne pas se valider en l'état, la puissance des évènements situationnels infléchissant les choix réalisés a priori par les enseignants.

Ces données amènent un nouveau regard sur certaines contributions (Rapport de recherche PIREF, 2007) qui ont bien mis en exergue qu'une pratique adressée à l'ensemble d'une classe provoque des effets différents : « elle s'avère profitable pour les bons élèves et les moyens et peu profitable ou pas du tout profitable pour les élèves faibles. Pour ces derniers, tout se passe comme s'ils ne pouvaient pas bénéficier d'une confrontation aux savoirs en jeu ». Or, nos conclusions montrent que cet état de fait n'est pas figé dans le temps et que les dynamiques différentielles peuvent se reconfigurer au service des apprentissages de tous. 


\section{eJRIEPS 42 juillet 2017}

\section{Conclusion}

II nous semble avoir mis en évidence dans cette recherche que les transitions codiques rendent compte d'un compromis situationnel entre des savoirs professionnels qui seraient appréhendés comme des prescriptions au moment de l'élaboration des contenus d'enseignement, et des situations interactives impliquant d'ingénieux ajustements dans les canaux de communication. Ces compromis conduisent à révéler que les transitions codiques évoluent en cours d'action dans le sens d'une rétrogénèse sémiotique pour les élèves les plus en difficultés, l'enseignant utilisant initialement le canal le plus abstrait (verbal) pour revenir à des canaux plus concrets par la mise en jeu de gestes et du corps. Ce changement rend compte de la relation conjointe entre activité de l'élève et activité de l'enseignant. Resitué dans notre cadre théorique, ce retour sur hypothèse dévoile que les indicateurs que nous avons choisis sont pertinents pour décrire les phénomènes topogénétiques, mésogénétiques et chronogénétiques inhérents au contrat-milieu didactique, mais nécessitent une articulation de deux échelons d'analyse, micro et mésodidactique. Ainsi, nous sommes invités à penser que le contexte de l'institution scolaire avec ses contraintes, dans cette discipline et pour cette activité, et l'épistémologie pratique des enseignants sollicités pour cette étude constituent un empêchement, au sens didactique, d'une mise en jeu immédiatement opérationnelle des canaux de communications. Ce processus conduit les professeurs à intuitivement alterner les canaux de communication afin de gérer la dynamique différentielle du doublet contrat-milieu. Sans doute s'agit-il d'une compétence professionnelle à prendre en compte dans la formation des enseignants d'EPS, plus particulièrement au moment de la construction des dispositifs d'étude qu'ils vont proposer aux élèves.

\section{Références}

Amade-Escot, C., Verscheure, I. \& Devos-Prieur, O. (2002). Agencement des milieux et régulations didactiques: outils d'analyse de l'activité du professeur en éducation physique. Les dossiers des sciences de l'éducation, 8, 87-97.

Anzieu, D. \& Cosnier, J. (1982). Les voies du langage. Paris : Dunod.

Astolfi, JP. \& Develay, M. (1989). Didactique des sciences. Paris : Presses Universitaires de France.

Bardin, L. (1991). L'analyse de contenu. Paris: PUF.

Bataille, A. (2011). Les modes de communication, une pédagogie innovante pour les enfants polyhandicapés. Toulouse : Erès « trame». 


\section{eJRIEPS 42 juillet 2017}

Barrière-Boizumault, N. (2013). Les communications non verbales des enseignants d'Education Physique et Sportive: formes et fonctions des CNV, croyances et réalisation effective des enseignants, ressenti des effets par les élèves. Thèse de Doctorat STAPS, Université Claude Bernard, Lyon 1.

Boizumault, N. \& Cogérino, G. (2012). La mise en scène corporelle de l'enseignant d'EPS : les communications non verbale au service de l'efficacité de l'enseignant. Staps, 98, 67-79.

Boudard, JM. \& Robin, JF. (2012/1). Pratique et régulation didactique en Education Physique et Sportive et place des savoirs techniques : illustration à travers une étude de cas. Staps, 95, 23-41.

Brière-Guenoun, F. (2014). Instruire les gestes didactiques de métier. Contribution à un programme de recherche en didactique comparée à partir d'études menées en Education Physique et Sportive. Habilitation à Diriger les Recherches, Université Toulouse Jean-Jaurès.

Broussal, D. \& Bucheton, D. (2008). Interagir en début de cours : les enjeux didactiques et discursifs. Revue Education \& Didactique, 2(3), 59-75.

Brousseau, G. (1998). Théorie des situations didactiques. Grenoble : La pensée sauvage.

Brousseau, G. (2004). Tâche, situation, activité.

Brown, R. (2000). Social identity theory, past achievements, current problems, and future challenges. Europeen journal of social psychology, 30, 745-778.

Catteau, R. (1992). La natation de demain. Biarritz : Atlantica.

Chevallard, Y. (1997). Familière et problématique, la figure du professeur. Recherche en didactique des mathématiques, 17(2). 17-54

Cosnier, J. \& Brossard, A. (1984). La communication non verbale. Paris, Neuchâtel: Delachaux \& Niestlé.

Dijksterhuis, A. \& Nordgren, L.F. (2006). A theory of unconscious thought. Perspectives on Psychological Science, 1, 95-109.

Durand, M. (2001). L'enseignement de l'EPS comme action située ; proposition pour une approche d'anthropologie cognitive. Staps, 55, 79-100.

Evin, A., Sève C. \& Saury, J. (2013). Activité de l'enseignant et dynamique coopérative au sein de dyades d'élèves. une étude de cas dans des tâches d'escalade en Education Physique. Recherches en Education, 15, 109-119.

Famose, J.P. (1983). Stratégies pédagogiques, tâches motrices et traitement de l'information. Dans JP. Famose, J. Bertsch, E. Champion \& M. Durand, Tâches 


\section{eJRIEPS 42 juillet 2017}

motrices et pédagogiques en Education Physique et Sportive (pp. 9-21). Paris: Revue EPS

Gal, N. (1993). Savoir nager, de l'école aux associations. Paris : Revue EPS.

Leplat, J. (2000). L’analyse psychologique de l'activité en ergonomie. Toulouse : Octares.

Leutenegger, F. (2000). Construction d'une « clinique » pour le didactique. Une étude des phénomènes temporels de l'enseignement. Recherche en Didactique des Mathématiques, 20(2), 209-250.

Leutenegger, F. (2008). Entrée dans le code de l'écrit à l'école enfantine et articulation entre le collectif et l'individuel: comparaison de deux études de cas. Education \& Didactique, 2(2), 7-42.

Leutenegger, F. (2009). Le temps d'instruire. Approche clinique et expérimentale du didactique ordinaire en mathématiques. Bruxelles : Peter Lang.

Ligozat, F. (2008). Un point de vue de Didactique comparée sur la classe de mathématiques. Etude de l'action conjointe $d u$ professeur et élèves à propos de l'enseignement/apprentissage de la mesure de grandeurs dans des classes françaises et suisses romandes. Thèse de doctorat en sciences de l'éducation, FPSE, Université de Genève \& U.F.R. Psychologie et sciences de l'éducation, Université de Provence.

Loquet, M., Garnier, A. \& Amade-Escot, C. (2002). Transmission des savoirs dans des institutions différentes : enseignement scolaire, entraînement sportif, transmission chorégraphique, Revue Française de Pédagogie, 141, 99-109.

Loquet, M., Roessle, S. \& Roncin, E. (2007). L'action conjointe dans le système didactique en APSA, les formes non verbales de la communication didactique. Dans G. Sensevy \& A. Mercier, Agir ensemble. L’action conjointe du professeur et des élèves dans le système didactique (pp. 123-151). Rennes : PUR.

Mahut, B. (2003). Approche sémiotique des interactions didactiques, geste et verbe en situation didactique. Thèse de doctorat en sciences du langage, Université de Besançon.

Mahut, B., Mahut, N. Gréhaigne, J-F. \& Masselot, M. (2005). Gestuelle de l'enseignant en natation et coconstruction de sens. Science \& Motricité, 56, 43-63.

Magne, P. \& Schmitt, A. (2002). Réussir et comprendre les activités aquatiques. Nantes: CRDP pays de la Loire.

McNeil, D., (1992). Hand and mind: What gesture reveal about thought. Chicago : University of Chicago Press. 


\section{eJRIEPS 42 juillet 2017}

Ministère de l'Education nationale. Note de service $n^{\circ}$ 94-116 du 9 mars 1994. Sécurité des élèves. Pratique des activités physiques scolaires. Bulletin officiel n¹1 du 17 mars 1994.

Ministère de l'Education nationale. Circulaire $n^{\circ 2004-138 ~ d u ~} 13$ juillet 2004. Les risques particuliers à l'enseignement de l'EPS et au sport scolaire. Bulletin officiel n`32 du 9 septembre 2004.

Ministère de l'Education nationale. Arrêté du 8 juillet 2008. Programme de l'enseignement d'éducation physique et sportive pour les classes de sixièmes, de cinquième, de quatrième et de troisième du collège. Bulletin officiel spécial nº du 28 août 2008.

Ministère de l'éducation nationale. Circulaire n²011-090 du 7 juillet 2011. Natation, enseignement dans les premier et second degrés. Bulletin officiel n²8 du 14 juillet 2011.

Moulin, JF. (2004). Le discours silencieux du corps enseignant. La communication non verbale du maître dans les pratiques de classe. Carrefour de l'éducation, 17, 142159.

Pain, J. (2012). Pratiques de classe et autorité. Recherche et Formation, 71(3), 99-112.

Pelayo, P. (1999). La natation au collège et au lycée. Paris : Revue EPS.

Rilhac, P. (2007). Actions de l'élève et milieux didactiques: la notion de « surassujetissement ». Carrefours de l'éducation, 24, 159-182.

Roesslé, S. \& Loquet, M. (2005). Etude des gestes didactiques lors des interactions parents/enfants dans l'activité aquatique d'éveil. Dans N. Benguigui, P. Fontayne, M. Desbordes \& B. Bardy, Recherches actuelles en Sciences du Sport. Actes du $9^{\text {ème }}$ congrès international de l'ACAPS, p.613-614.

Roncin, E. \& Loquet, M. (2007). Enseigner la danse à des adolescents avec autisme, l'ingéniosité des gestes enseignants. 4ème biennale ARIS 2006, Coconstruire des savoirs la recherche et les métiers de l'intervention dans les APSA. Besançon.

Saujat, F. (2004). Comment les enseignants débutants entrent dans le métier. Formation et pratiques d'enseignement, 1, 97-106.

Schubauer-Leoni, ML. (1997). Interaction didactique et interactions sociales: quels phénomènes et quelles constructions conceptuelles . Skolé 7, 103-134.

Schubauer-Leoni, ML. \& Leutenegger, F. (2002). Expliquer et comprendre dans une approche clinique/expérimentale du didactique ordinaire. Dans F. Leutenegger, \& M. Saada-Roberts, Expliquer et comprendre en science de l'éducation (pp. 227-251). Paris, Bruxelles : De Boeck. 


\section{eJRIEPS 42 juillet 2017}

Sensevy, G. (2001). Théorie de l'action et action de professeur. Dans J-M. Beaudouin, \& J. Friedrich (eds). Théories de l'action et éducation (pp. 203-224). Bruxelles: De Boeck, Raisons Educatives.

Sensevy, G. (2007) (dir.). Rapport de recherche PIREF. Caractérisation des pratiques d'enseignement et détermination de leur efficacité. La lecture et les mathématiques au cours préparatoire.

Sensevy, G. \& Mercier, A. (2007). Agir ensemble, action conjointe du professeur et des élèves dans le système didactique. Rennes : Presses Universitaires de Rennes.

Sensevy, G. \& Quilio, S. (2002). Le discours du professeur. Vers une pragmatique didactique. Revue Française de Pédagogie, 141, 47-56.

Theureau, J. (2010). Les entretiens d'autoconfrontation et de remise en situation par les traces matérielles et le programme de recherche "cours d'action». Revue d'anthropologie des connaissances, 4(2), 287-322.

Theureau, J. \& Jeffroy, F. (1994). Ergonomie des situations informatisées, la conception centrée sur le cours d'action de l'utilisateur. Toulouse : Octares.

Trottin, B. \& Cogérino, G. (2009). Filles et garçons et EPS : approche descriptive des interactions verbales entre enseignant et élève. Staps, 83, 69-85.

Vergnaud, G. (2007). Définition du concept de schème. Recherches en Education, 4, 1722. 